\title{
Bosentan and sildenafil: successful treatment in a sclerodermic patient with refractory ulcers
}

\author{
E. Catarsi ${ }^{1}$, M. Doveri ${ }^{2}$, A. Tavoni ${ }^{1}$ \\ 'U.O. Immunoallergologia, Ospedale S. Chiara, Pisa, Italy; \\ ${ }^{2}$ U.O. Reumatologia, Ospedale S. Chiara, Pisa, Italy
}

\begin{abstract}
SUMMARY
Systemic sclerosis is an inflammatory disease of the connective tissue characterized by vasculopathy and accumulation of collagen and other components of the connective matrix, affecting the skin and internal organs. The appearance of skin ulcers as a result of vascular damage is very common in the history of the disease. Skin ulcers, painful and slow healing due to atrophy and local ischemia, get worse the quality of life of patients. Often, the use of conventional therapies (such as calcium channel blockers and prostanoids) does not cause the complete healing of the lesions.

We report the case of a patient in whom therapeutic association between endothelin antagonist (bosentan) and phosphodiesterase-V inhibitor (sildenafil) resulted in complete healing of old ulcers both to upper and lower limbs and allowed the interruption of intravenous therapies.
\end{abstract}

Key words: Bosentan, Sildenafil, Ulcer, Sistemic sclerosis.

Reumatismo, 2013; 65 (2): 79-81

\section{INTRODUCTION}

ystemic sclerosis is a rare disease, characterized by fibrosis and vasculopathy, with variable internal organ involvement. The etiology is still not fully elucidated, but the pathophysiology is characterized by immunologic mechanisms, vascular endothelial cell injury and excessive activation of fibroblasts. Skin is very often involved. Digital ulcerations are characterized by severe pain, tissue loss, impaired hand function, and a deep impact on quality of life. The healing is slow due to atrophic, fibrotic, and avascular nature of the local tissue. We reported our experience in the treatment of a sclerodermic patient with inveterate ulcers refractory to the conventional treatment.

\section{CASE REPORT}

In 1988 a 57-year old woman presented for the first time to our attention. The patient denied history of smoking and dys- lipidemia; she reported regular menstrual cycles, and menopause at the age of 50 .

At the time of observation she referred from few months the appearance of Raynaud's phenomenon (RP) of the hands, cutaneous thickness of the face, neck and upper limbs, arthromyalgia, sicca syndrome. The overall evaluation with instrumental and laboratory tests showed positivity for antinuclear and antitopoisomerase- 1 antibodies, esophageal hypotonia and hypokinesia, active scleroderma-pattern at the capillaroscopy. The diagnosis of scleroderma was made and the patient started treatment with calcium antagonists, antiplatelets agents and low-dose of corticosteroids. In 1997 she was hospitalized for the appearance of digital ulcers in order to start intravenous therapy with prostanoids. A high-resolution computed tomography performed in that period highlighted for the first time areas of honey-combing.

In the following years the patient was periodically hospitalized to perform revaluation of disease and to treat ulcers. Ulcers were always acral, often infected, both up-
Corresponding author: Eleonora Catarsi U.O. Immunoallergologia Ospedale Santa Chiara Via Roma, 67 - Pisa, Italy E-mail: eleonoracatarsi@hotmail.com 


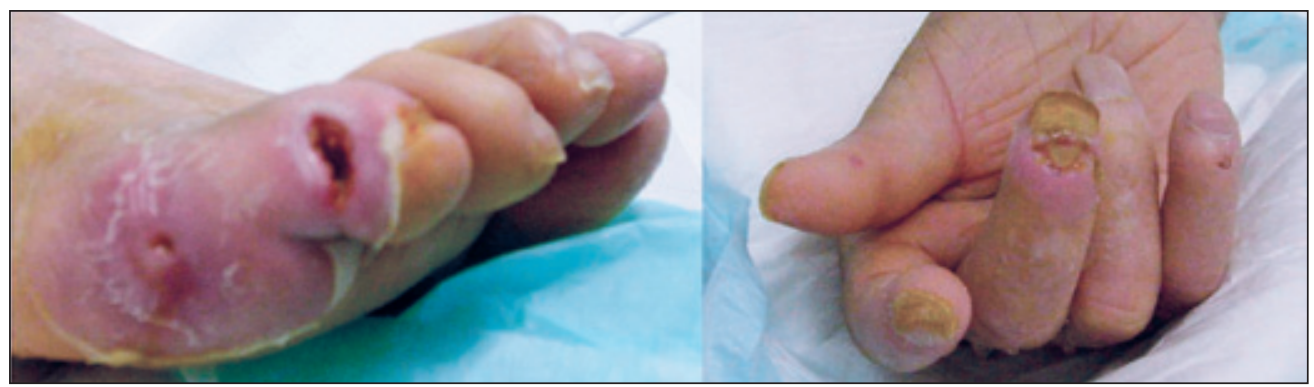

Figure 1 - Ulcer of the $\mathrm{V}$ finger of the right foot and ulcer of the III finger of the right hand (November 2007).

per and lower limbs. They had a course of seasonality and a latency of healing variable from 2 to 8 months. In every occasion the patient was treated with local dressings, hyperbaric oxygen therapy, platelet gel and intravenous prostacyclins. The therapy with prostanoids has always been performed in a discontinuous manner because of the poor tolerance of the drug by the patient. Over the years, instrumental tests have always shown stable organ involvement. No signs of pulmonary hypertension or affection of large vessels were detected.

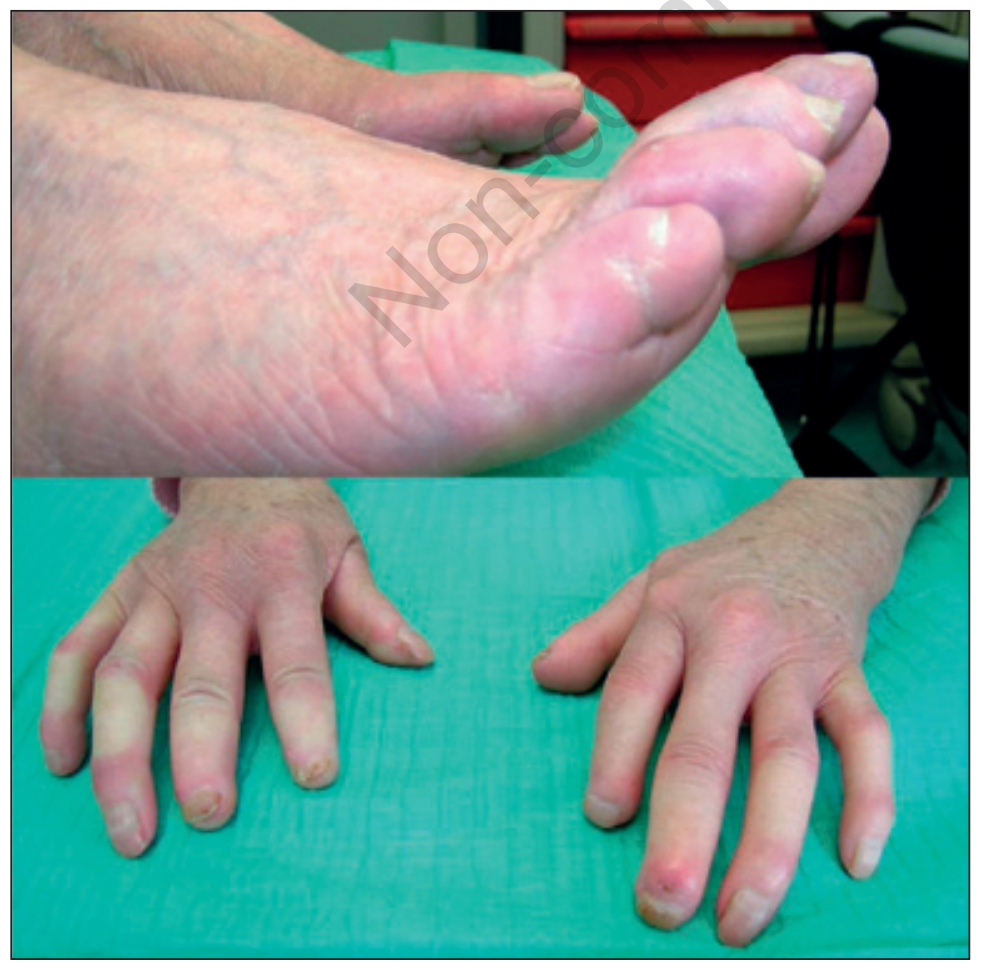

Figure 2 - Complete healing of the ulcers (February 2011).
In September 2006, for the appearance of new ulcers (I and II finger of right hand, II finger of the left hand and V finger of left foot) resistant to conventional therapies until taken, the patient started therapy with bosentan (62.5 mg twice a day, increasing to $125 \mathrm{mg}$ twice a day). The patient refused prostanoids therapy.

In the following year, the patient did not present new ulcers and she obtained the complete healing of the ulcers of the right hand (lesions in the left hand and left foot remained active).

In November 2007, for the appearance of four new ulcers (I and V finger of the right foot, III finger of the hands) (Fig. 1) the patient started therapy with sildenafil $25 \mathrm{mg}$ daily and discontinued bosentan.

In May 2008, for lack of improvement of pre-existing ulcers and appearance of a new ulcer (I finger of the right hand) bosentan was reintroduced until the full dose and sildenafil was increased to $30 \mathrm{mg}$ daily.

In the two following years the patient showed progressive improvement of ulcers by reducing the size of some and complete recovery of others.

In November 2008 the patient had only three ulcers (II finger of right hand, III finger of left hand and $\mathrm{V}$ finger of the right foot).

In May 2010, the patient still had two ulcers of the hands, although on the road to recovery.

From February 2011 to now, the patient no longer presented skin ulcers (Fig. 2). She continues therapy with bosentan, sildenafil, low-dose of corticosteroids, antiplatelets and calcium antagonists. 


\section{DISCUSSION}

The analysis of this case shows that the combination of an endothelin antagonist with a phosphodiesterase- $\mathrm{V}$ inhibitor may exhibit many advantages such as the healing of old digital ulcers, both to upper and lower limb even in the absence of therapy with intravenous prostanoids and in those cases in which conventional therapies (such as calcium antagonists) failed (1-3). Other advantages are the effectiveness over the time, the good tolerability and the safety profile. The European League Against Rheumatism (EULAR) and the EULAR Scleroderma Trials And Research (EUSTAR) Group recommendations of 2009 (3) for the treatment of systemic sclerosis- related digital vasculopathy only suggest the use of calcium antagonists, prostanoids and bosentan. In particular it is emphasized that the use of the first two drugs reduces the frequency and the severity of the RP; the dihydropyridinetype calcium antagonists are recommended as first-line therapy for RP and prostanoids are indicated for the treatment of severe RP. Prostanoids are also efficacious in healing digital ulcers so they should be considered in the treatment of active digital ulcers. The use of bosentan is recommended after failure of treatment with calcium antagonists and prostanoids. It is also pointed out that this drug is no efficacious in the treatment of active digital ulcers but only in the prevention of new ulcers. The use of phosphodiesterase- $\mathrm{V}$ inhibitors is not currently suggested. Some work in the literature report the efficacy of therapy with phosphodiesterase- $\mathrm{V}$ inhibitors in healing of digital ulcers in systemic sclerosis (4-9). Few experiences are reported on the effectiveness of the association of phosphodiesterase- $\mathrm{V}$ inhibitors and bosentan (10-12) even though results are similar to those we have described. Given the incidence of vascular disease in systemic sclerosis, and the potential possibility to limit the use of intravenous therapies, it is desirable to do other studies in order to prove or disprove the efficacy of the association between endothelin antagonists and phosphodiesterase- $\mathrm{V}$ inhibitors in the treatment of digital ulcers in systemic sclerosis.

\section{REFERENCES}

1. Sicińska J, Rudnicka L. Current treatment of systemic sclerosis. Part II. Vascular and antifibrotic treatment. Pol Merkur Lekarski. 2008; 25: 196-200. (Article in Polish).

2. Meyer MF, Daigeler A, Lehnhardt M, et al. Therapeutic management of acral manifestations of systemic sclerosis. Med Klin (Munich). 2007; 102: 209-18. (Article in German).

3. Kowal-Bielecka O, Landewé R, Avouac J, et al. EULAR recommendations for the treatment of systemic sclerosis: a report from the EULAR Scleroderma Trials and Research group (EUSTAR). Ann Rheum Dis. 2009; 68: 620-8.

4. Kumar U, Gokhle SS, Sreenivas V, et al. Prospective, open-label, uncontrolled pilot study to study safety and efficacy of sildenafil in systemic sclerosis-related pulmonary artery hypertension and cutaneous vascular complications. Rheumatol Int. 2013; 33: 1047-52.

5. Brueckner CS, Becker MO, Kroencke T, et al. Effect of sildenafil on digital ulcers in systemic sclerosis: analysis from a single centre pilot study. Ann Rheum Dis. 2010; 69: 1475-8.

6. Cairoli E, Garra V, Martínez M. Sildenafil in the treatment of digital ulcers in patients with systemic sclerosis. Actas Dermosifiliogr. 2009; 100: 830-2.

7. Friedrichson E, Rehberger P, Fuhrmann JT, et al. Fast and efficient healing of sclerodermaassociated acral ulcers with sildenafil. Hautarzt. 2008; 59: 230-2. (Article in German).

8. Colglazier CL, Sutej PG, O'Rourke KS. Severe refractory fingertip ulcerations in a patient with scleroderma: successful treatment with sildenafil. J Rheumatol. 2005; 32: 24402.

9. Della Rossa A, Doveri M, D'Ascanio A, et al. Oral sildenafil in skin ulcers secondary to systemic sclerosis. Scand J Rheumatol. 2011; 40: 323-5.

10. Moinzadeh P, Hunzelmann N, Krieg T. Combination therapy with an endothelin-1 receptor antagonist (bosentan) and a phosphodiesterase $\mathrm{V}$ inhibitor (sildenafil) for the management of severe digital ulcerations in systemic sclerosis. J Am Acad Dermatol. 2011; 65: e102-4.

11. Ambach A, Seo W, Bonnekoh B, Gollnick H. Low-dose combination therapy of severe digital ulcers in diffuse progressive systemic sclerosis with the endothelin-1 receptor antagonist bosentan and the phosphodiesterase $\mathrm{V}$ inhibitor sildenafil. J Dtsch Dermatol Ges. 2009; 7 : 888-91.

12. Opitz C, Klein-Weigel PF, Riemekasten G. Systemic sclerosis - a systematic overview: part 2 - immunosuppression, treatment of SSc-associated vasculopathy, and treatment of pulmonary arterial hypertension. Vasa. 2011; 40: $20-30$. 